\title{
Efectos de la inmunoesterilización sobre la ganancia de peso, rendimiento de carcasa y peso de vellón en carnerillos Junín
}

\author{
Effects of immunosterilization on body weight gain, carcass yield and fleece \\ weight in young Junin rams
}

Ide Unchupaico P. ${ }^{1}$, Carlos Quispe E. ${ }^{1,3}$, Gerson Flores M. ${ }^{1}$, Edith Ancco G. ${ }^{2}$

\section{Resumen}

El objetivo del estudio fue evaluar el efecto de la inmunoesterilización mediante la aplicación de una proteína sintética anti-GnRH sobre la ganancia de peso, rendimiento de carcasa y peso de vellón en ovinos Junín. El estudio fue conducido en la SAIS «Túpac Amaru», Junín, Perú, a una altitud de 3587 msnm entre setiembre y diciembre de 2017. Se utilizaron 80 carnerillos de 11-13 meses y se distribuyeron al azar en cuatro tratamientos (20 por grupo). Los tratamientos fueron T1: castración quirúrgica, T2 y T3: aplicación de 0.5 y $1.0 \mathrm{ml}$ de vacuna anti-GnRH, y T4: control (aplicación de un placebo). Para la inmunoesterilización se utilizaron dos dosis de Improvac ${ }^{\circledR}$ vía subcutánea a intervalos de 21 días. El peso vivo se tomó cada 15 días durante tres meses y el peso de vellón sucio se tomó inmediatamente después de la esquila. Los resultados muestran que la aplicación de la vacuna anti-GnRH a una dosis de $1.0 \mathrm{ml}$ mejora los incrementos de peso diario y el peso vivo final $(\mathrm{p}<0.05)$, mientras que para el rendimiento de carcasa y peso de vellón no se hallaron diferencias significativas entre tratamientos. Se concluye que la aplicación de la vacuna anti-GnRH en ovinos mejora el rendimiento productivo de estos animales.

Palabras clave: carnerillos; inmunoesterilización; GnRH; castración; carcasa; Improvac

\section{AbSTRaCT}

The aim of the study was to evaluate the effect of immune sterilization on body weight gain, carcass yield and fleece weight in Junin sheep by applying an anti-GnRH synthetic protein. The study was conducted at the SAIS «Tupac Amaru», Junin, Peru, at

${ }^{1}$ Laboratorio de Reproducción Animal, Facultad de Zootecnia, Universidad Nacional del Centro del Perú, Junín, Perú

${ }^{2}$ Estación Experimental El Mantaro, Facultad de Zootecnia, Universidad Nacional del Centro del Perú, Junín, Perú

${ }^{3}$ E-mail: cquispe.vet@gmail.com

Recibido: 5 de marzo de 2018

Aceptado para publicación: 31 de agosto de 2018 
an altitude of $3587 \mathrm{~m}$ between September and December 2017. Eighty 11-13-month-old rams were used and randomly distributed into four treatments (20 per group). Treatments were T1: surgical castration, T2 and T3: application of 0.5 and $1.0 \mathrm{ml}$ of anti-GnRH vaccine, and T4: control (application of a placebo). For the immune sterilization, two doses of Improvac ${ }^{\circledR}$ were subcutaneously applied in the axillary region at 21-day intervals. Body weight was taken every 15 days for three months and the weight of dirty fleece was taken immediately after shearing. The results show that the application of anti-GnRH vaccine at a dose of $1.0 \mathrm{ml}$ improvement increases daily body weight gain and the final body weight $(p<0.05)$, while for carcass yield and fleece weight no significant differences were found between treatments. It is concluded that the application of the anti-GnRH vaccine in sheep improves the productive performance of these animals.

Key words: young rams; immuno sterilization; GnRH; castration; carcass; Improvac

\section{INTRODUCCIÓN}

La mayor actividad ganadera del país se desarrolla en praderas altoandinas donde los sistemas de producción animal varían en función de factores ambientales, tipo de forraje, y tamaño del predio. Las especies más importantes corresponden a los vacunos, ovinos, y camélidos como fuente de carne, leche, lana y fibra, y que en la mayoría de los casos representan la única fuente de ingreso económico (Flores y Oscanoa, 1992). La región Junín ha evidenciado una reducción de $29 \%$ en la población ovina al 2012 , de acuerdo con el último censo agropecuario (CENAGRO, 2012).

Los llamados métodos contraceptivos pueden ser quirúrgicos, donde se considera la extracción de tejido reproductivo (Slatter, 2003) y no quirúrgicos donde se utilizan sustancias que inhiben los procesos de la función reproductiva (von Borell et al., 2009). Estos últimos incluyen la terapia hormonal, el uso de antagonistas de GnRH o castración química, cuya diferencia es el modo de acción en el organismo, y las técnicas que destruyen tejido mediante el uso del elastrador, emasculador y ligantes (Oliveira et al., 2012).
La hormona liberadora de gonadotropinas $(\mathrm{GnRH})$ es un péptido que se libera a través de neuronas secretoras del hipotálamo y es responsable de la liberación de la hormona folículo estimulante (FSH) y de la hormona luteinizante (LH). La primera actúa a nivel de las células de Sertolli y la segunda a nivel de las células de Leydig para la producción de testosterona y la consecuente producción de espermatozoides (Amann, 1983). La GnRH es un elemento clave en la cascada endocrina que apoya el crecimiento, funcionalidad y maduración de los testículos (Amann y Schanbacher, 1983).

Los péptidos antagonistas naturales de la GnRH regulan la función testicular (Mihsler et al., 2016). La inmunización contra la GnRH disminuye la liberación de LH, FSH y testosterona. Los agonistas de GnRH de acción prolongada disminuyen los receptores de GnRH (King y Millar, 1995). Actúan como una vacuna que estimula el sistema inmunitario para producir anticuerpos específicos frente a la $\mathrm{GnRH}$, lo cual inhibe temporalmente la función testicular. El empleo de dos dosis con intervalo de 7-10 días produce una castración inmunológica en el animal (Brown et al., 1995). 
Se dispone de escasos estudios en ovinos sobre las implicancias de la inmunoesterilización usando vacunas antagonistas de la GnRH. En la mayoría de las especies se recomienda usar esta vacuna en la etapa prepuberal (Deaver et al., 1988), donde la frecuencia de episodios secretores de $\mathrm{GnRH}$ aumenta (Rodriguez y Wise, 1989) debido al desarrollo intenso del tejido testicular. En ovinos de doble propósito de la sierra peruana la pubertad se inicia a partir de los 4-7 meses, dependiendo del manejo, nutrición, sanidad, genética, clima, etc. (Aliaga y Calle, 1995).

Se ha demostrado que su uso mejora características productivas como calidad de carne, rendimiento de carcasa, peso vivo y disminuye la agresividad en cuyes y porcinos (Osorio, 1999; Terán, 2015). Bajo ese contexto, el objetivo del presente estudio fue evaluar el efecto de la administración de un inmunoesterilizador sobre variables productivas de importancia en ovinos.

\section{Materiales y Métodos}

El presente estudio se llevó a cabo en la Unidad de Producción de Pachacayo, perteneciente a la SAIS Túpac Amaru Ltda. N. ${ }^{\circ}$ 1, ubicado en la región Junín, distrito de Canchayllo, provincia de Jauja, a una altitud promedio de $3587 \mathrm{msnm}$, con una temperatura ambiental promedio de $8{ }^{\circ} \mathrm{C}$ y una humedad relativa promedio de $70 \%$. El estudio se realizó entre setiembre y diciembre de 2017.

Se utilizaron 80 carnerillos en óptimo estado de salud, pertenecientes al grupo de majada, cuyo rebaño principal estaba constituida por 250 carnerillos entre 11 y 13 meses de edad. El área de pastoreo fue un pajonal de condición buena, dominado por Festuca humilior y Calamagrostis vicunarum. Los animales fueron distribuidos al azar en cuatro tratamientos a razón de 20 animales por grupo de estudio. Los tratamientos fueron T1: castración quirúrgica, T2: aplicación de 0.5 $\mathrm{ml}$ de vacuna anti-GnRH, T3: aplicación de $1.0 \mathrm{ml}$ de vacuna anti-GnRH, T4: control, con aplicación de un placebo. La inmunoesterilización se hizo con el producto comercial Improvac ${ }^{\circledR}$ (Zoetis) mediante dos aplicaciones subcutáneas en la región axilar con un intervalo de 21 días (Pfizer, 2009). La castración quirúrgica en $\mathrm{T} 1$ fue por orquiectomía bilateral (Howe, 2006). Improvac $\AA$ solo se comercializa para cerdos, de allí que para las dosis empleadas se consideró que el peso de los carnerillos era $30 \%$ menor que cerdos de esa edad.

Se registró el peso vivo cada 15 días por tres meses con una balanza calibrada tipo jaula, con capacidad hasta $100 \mathrm{~kg}$. El peso de vellón sucio se tomó inmediatamente después de la esquila de los animales previo al beneficio, utilizando una balanza tipo reloj con capacidad de hasta $5 \mathrm{~kg}$. El análisis estadístico se realizó mediante estadística descriptiva y análisis de varianza, previa prueba de normalidad para verificar la distribución normal de los datos. La comparación de medias se realizó con una prueba de Tukey a un nivel de 0.05 de significancia. Adicionalmente, se utilizó una prueba de contingencia para variables no paramétricas (Calzada, 1982). El paquete estadístico utilizado fue SAS v. 9.0.

\section{Resultados}

En el Cuadro 1 se muestran los efectos de los tratamientos sobre la ganancia de peso, rendimiento de carcasa y peso de vellón. El empleo de la dosis de $1.0 \mathrm{ml}$ del inmunoesterilizador presentó mejores resultados en términos de peso final, ganancia de peso por día y peso de carcasa con relación al grupo control $(\mathrm{p}<0.05)$; sin embargo, no hubo diferencia significativa para incremento de peso total, el rendimiento de carcasa y el peso de vellón. El rendimiento productivo entre T2 (Improvac $0.5 \mathrm{ml}$ ) y el control fue estadísticamente similar. Por otro lado, la castración quirúrgica tuvo los menores rendimientos, por debajo del grupo control. 
Cuadro 1. Efecto de la castración quirúrgica e inmunoesterilización sobre variables productivas en carnerillos de la raza Junín ( $\mathrm{n}=20$ por tratamiento)

\begin{tabular}{lcccc}
\hline \multirow{2}{*}{ Variables } & $\begin{array}{c}\text { T1 } \\
\text { Castración } \\
\text { quirúrgica }\end{array}$ & $\begin{array}{c}\text { T2 } \\
0.5 \mathrm{ml} \mathrm{de} \\
\text { Improvac } ®\end{array}$ & $\begin{array}{c}\text { T3 } \\
1.0 \mathrm{ml} \mathrm{de} \\
\text { Improvac }{ }^{\circledR}\end{array}$ & $\begin{array}{c}\text { T4 } \\
\text { Control }\end{array}$ \\
\hline Peso inicial $(\mathrm{kg})$ & $28.0 \pm 3.0^{\mathrm{a}}$ & $27.0 \pm 2.4^{\mathrm{a}}$ & $28.3 \pm 2.4^{\mathrm{a}}$ & $27.4 \pm 1.8^{\mathrm{a}}$ \\
Peso final $(\mathrm{kg})$ & $30.1 \pm 2.8^{\mathrm{c}}$ & $32.0 \pm 2.3^{\mathrm{bc}}$ & $35.4 \pm 2.9^{\mathrm{a}}$ & $32.8 \pm 2.8^{\mathrm{b}}$ \\
Ganancia de peso/día (g) & $22.9 \pm 22.7^{\mathrm{c}}$ & $58.3 \pm 21.1^{\mathrm{b}}$ & $80.3 \pm 40.0^{\mathrm{a}}$ & $59.3 \pm 30.4^{\mathrm{b}}$ \\
Incremento total $(\mathrm{kg})$ & $2.1 \pm 2.1^{\mathrm{b}}$ & $5.2 \pm 1.9^{\mathrm{a}}$ & $6.5 \pm 3.2^{\mathrm{a}}$ & $5.4 \pm 2.7^{\mathrm{a}}$ \\
Peso carcasa $(\mathrm{kg})$ & $14.0 \pm 1.4^{\mathrm{b}}$ & $15.1 \pm 0.8^{\mathrm{b}}$ & $16.6 \pm 1.7^{\mathrm{a}}$ & $15.0 \pm 1.3^{\mathrm{b}}$ \\
Rendimiento de carcasa (\%) & $46.0 \pm 1.7^{\mathrm{a}}$ & $46.1 \pm 1.6^{\mathrm{a}}$ & $46.7 \pm 2.7^{\mathrm{a}}$ & $45.9 \pm 1.6^{\mathrm{a}}$ \\
Peso de vellón $(\mathrm{kg})$ & $1.3 \pm 0.2^{\mathrm{a}}$ & $1.3 \pm 0.2^{\mathrm{a}}$ & $1.3 \pm 0.2^{\mathrm{a}}$ & $1.4 \pm 0.2^{\mathrm{a}}$ \\
\hline
\end{tabular}

$a, b, c$ Letras diferentes dentro de filas indican diferencias entre tratamientos $(p<0.05)$

\section{Discusión}

El efecto del antagonista de GnRH a una dosis de $1.0 \mathrm{ml}$ se debió a la inhibición de la función testicular que tiene la vacuna, no permitiendo que la GnRH actúe sobre las células de Leydig para producción de espermatozoides, de modo que parte de la energía destinada para este fin sería desviada a la deposición de grasa; sin embargo, esa ruta aún no ha sido del todo investigada (Tilbrook y Clarke 2001).

La información existente para ovinos es bastante incierta. Osorio et al . (1999) obtuvo diferencias entre ovinos inmunoesterilizados con relación a los quirúrgicamente castrados para rendimiento de carcasa, aspecto que no fue significativo en el presente estudio; así mismo, Ripoll (2016) reporta una reducción del ritmo de crecimiento y de la ganancia de peso en terneros, pero un aumento en la terneza y el porcentaje de veteado del lomo, y evita el deterioro del color de la carne en terneros pesados. El menor rendimiento obtenido en ovinos castrados quirúrgicamente probablemente fue debido a la redirección de nutrientes para reducir el proceso inflamatorio y regeneración de tejidos, pues no se observaron alteraciones postoperatorias.

Entre los factores que pueden afectar los resultados se encuentra la dosis utilizada. Pfizer (2009) y Terán (2015) sugieren $2 \mathrm{ml}$ por dosis, Basulto et al. (2003) utilizó $1.0 \mathrm{ml}$, similar a la dosis efectiva del presente estudio, obteniendo los mismos resultados para peso vivo, pero en perros. La mayoría de trabajos han sido realizados en porcinos (Calderón, 2011; Vela, 2012; Cardelino, 2013) en dosis de $2 \mathrm{ml}$ y donde se puede apreciar que los incrementos de peso y la calidad de la carne son favorecidos largamente. Pareciera que a mayor dosis, el efecto de la antiGnRH potencia su efecto y se distribuye más rápidamente en el organismo manteniendo picos elevados del producto en el animal (Pfizer, 2009; Adams et al., 2011).

El peso de vellón se incluyó como variable en el presente estudio debido a que otros estudios afirman la correlación positiva que hay con respecto al peso vivo (Vargas et al., 2007; Lembeyea et al., 2014); sin embargo, no hubo significancia estadística entre los tratamientos. 


\section{ConClusiones}

La inmunoesterilización mediante la aplicación de una vacuna anti-GnRH a una dosis de $1.0 \mathrm{ml}$ favorece el incremento de peso vivo en carnerillos, pero no tiene efecto sobre el rendimiento de carcasa y peso de vellón.

\section{Agradecimientos}

Agradecimiento especial a la SAIS Túpac Amaru Ltda. N. ${ }^{\circ} 1$ por su valiosa colaboración en la logística y desarrollo de la presente investigación.

\section{Literatura Citada}

1. Adams TE, Daley CA, Adams BM, Sakurai H. 2011. Testis function and feedlot performance of bulls actively immunized against gonadotropinreleasing hormone: effect of implants containing progesterone and estradiol benzoate. J Anim Sci 71: 811-817. doi: 10.2527/1993.714811x

2. Aliaga J, Calle R. 1995. Parámetros reproductivos y productivos de ovinos tropicales. Lima, Perú: Universidad Nacional Agraria La Molina. $650 \mathrm{p}$.

3. Amann RP. 1983. Endocrine changes associated with onset of spermatogenesis in Holstein bulls. J Dairy Sci 66: 26062622. doi: 10.3168/jds.S0022-0302(83)82135-3

4. Amann RP, Schanbacher BD. 1983. Physiology of male reproduction. J Anim Sci 57(Suppl 2): 380-403.

5. Basulto $R$, Milanes C, Rojas A, Fuentes $F$, Izquierdo $N$, Bertot $J A$, Hernández H, et al. 2003. Efectos de la inmunización contra GnRH sobre la estructura y función testicular en perros adultos. Biotecnol Aplicada 20: 20-24.

6. Brown BW, Mattner PE, Carroll PA, Hoskinson RM, Rigby RDG. 1995. Immunization of sheep against GnRH early in life: effects on reproductive function and hormones in ewes. J Reprod Fertil 103: 131-135. doi: 10.1530/ jrf.0.1030131

7. Calderón D. 2011. Evaluación de la inmunocastración en relación a cerdos castrados quirúrgicamente en la raza Camboroguth. Tesis de Ingeniero Zootecnista. Riobamba, Ecuador: Escuela Superior Politécnica de Chimborazo. $88 \mathrm{p}$.

8. Calzada J. 1982. Métodos estadísticos para la investigación. $5^{\circ}$ ed. Lima, Perú: Ed Milagros. $245 \mathrm{p}$.

9. Cardelino $G$ 2013. Evaluación del índice de conversión y consumo diario de alimento en lechones de sitio II y sitio III en función de la utilización del inmuno castrador químico Improvac, Laboratorio Pfizer, a los 90 y 121 días de vida. Tesis de Ingeniería en Producción Agropecuaria. Buenos Aires, Argentina: Universidad Católica Argentina. $30 \mathrm{p}$.

10. [CENAGRO] Censo Nacional Agropecuario. 2012. Instituto Nacional de Estadística e Informática. IV Censo nacional agropecuario. Lima, Perú. [Internet]. Disponible en: http:// censos.inei.gob.pe/cenagro/tabulados/

11. Deaver DR, Glass JD, Grieger DM, Reeves JJ. 1988. Effects of estradiol on secretion of LH, hypothalamic function and testicular development in bull calves. Domest Anim Endocrin 5: 307-316.

12. Flores E, Oscanoa L. 1992. Avances en la ecología de la nutrición de ovinos, alpacas y llamas en el ecosistema de puna seca. Boletín Técnico UNALM 3: 2-11.

13. Howe LM. 2006. Surgical methods of contraception and sterilization. Theriogenology 66: 500-509. doi: 10.1016/ j.theriogenology.2006.04.005

14. King JA, Millar RP. 1995. Evolutionary aspects of gonadotropin-releasing hormone and its receptor. Cell Mol Neurobiol 15: 5-23. doi: 10.1007/ BF02069556

15. Lembeye F, Castellaro G, Magofke JC, Uribe H. 2014. Correlaciones fenotípicas entre características de im- 
portancia económica en ovinos Suffolk Down y Merino Precoz en la zona central de Chile. Arch Med Vet 46: 103-109.

16. Mihsler L, Wagner H, Wehrend A. 2016. Suppression of sexual activity and reproduction in male small ruminants. Tierarztl Prax G N 44: 171-178. doi: 10.15653/TPG-151145

17. Oliveira BAS, Rocha LM, Mól B, Valle GR. 2012. Métodos cirúrgicos e não cirúrgicos de contracepção masculina em cães. Sinapse Múltipla 1: 1-14.

18. Osório J, Osório M, Faria H, Jardim R, Esteves R. 1999. Morfología «in vivo», en la canal y características productivas y comerciales en corderos corriedales no castrados y castrados. En: XXV Jornadas Científicas y IV Internacionales de la Sociedad Española de Ovinotecnia y Caprinotecnia. Teruel, España.

19. Pfizer. 2009. Inmunocastración en cerdos. Pfizer presenta Improvac. Pfizer Sanidad Animal, Departamento Técnico. [Internet]. Disponible en: https:// www.engormix.com/porcicultura/ articulos/inmunocastracion-cerdospfizer-presenta-t28602.htm

20. Ripoll G. 2016. Efecto de la inmunocastración de terneros a dos pesos vivos sobre los parámetros productivos y la calidad de la carne. Tesis de Maestría. España: Universidad de Ávila. 122 p.

21. Rodríguez RE, Wise ME. 1991. Advancement of postnatal pulsatile luteinizing hormone secretion in the bull calf by pulsatile administration of gonadotropin-releasing hormone during infantile development. Biol Reprod 44: 432-439. doi: 10.1095/biolreprod44.3.432

22. Slatter DH. 2003. Textbook of small animal surgery. $3^{\text {rd }}$ ed. USA: Elsevier Saunders. $2713 \mathrm{p}$.

23. Terán J. 2015. Efecto de la inmunoesterilización como alternativa a la esterilización quirúrgica tradicional en diferentes parámetros hormonales en ovinos (Ovis aries) como modelo experimental animal. Tesis de Médico Veterinario y Zootecnista. Latacunga, Ecuador: Universidad Técnica de Cotopaxi. $119 \mathrm{p}$.

24. Tilbrook AJ, Clarke IJ. 2001. Negative feedback regulation of the secretion and actions of gonadotropin-releasing hormone in males. Biol Reprod 64: 735742. doi: 10.1095/biolreprod64.3.735

25. Vargas F, Pérez M, De Lucas J. 2007. Evaluación preliminar de la profundidad y del área del Longisimus dorsi mediante uso de ultrasonografía y del rendimiento de la canal en ovinos de pelo En: XX Reunión de la Asociación Latinoamericana de Producción Animal (ALPA). Cusco, Perú.

26. Vela A. 2012. Efecto de la inmunocastración y castración quirúrgica en los parámetros productivos de cerdos. Tesis de Ingeniero Zootecnista. Riobamba, Ecuador: Escuela Superior Politécnica de Chimborazo. $38 \mathrm{p}$.

27. von Borell E, Baumgartner J, Giersing M, Jäggin N, Prunier A, Tuyttens FA, Edwards SA. 2009. Animal welfare implications of surgical castration and its alternatives in pigs. Animal 3: 1488-1496. doi: $10.1017 / \mathrm{S} 1751731109004728$ 